\title{
Rentabilidade e Eficiência no Setor Bancário Brasileiro
}

\author{
Paulo Sergio Ceretta \\ Carlos Alberto Pittaluga Niederauer
}

\section{RESUMO}

As transformações na economia internacional vêm atingindo em grande escala o setor bancário brasileiro. Para verificar o nível de competição no setor, e para investigar o desempenho comparativo entre os bancos brasileiros, aplicou-se o método da análise por envoltória de dados. Foram utilizados dados sobre o montante de capital dos proprietários, capital de terceiros, receita total e resultado do semestre de 144 instituições bancárias do país. Os bancos foram agrupados em três categorias: grande, médio e pequeno porte. Foi utilizada a abordagem da matriz BCG para enquadrar os diversos indicadores de eficiência operacional e de rentabilidade. Os resultados sugerem que os bancos de grande porte são os de melhor desempenho e os de pequeno porte os de pior desempenho.

Palavras-chaves: desempenho; análise por envoltória de dados; setor bancário.

\begin{abstract}
The Brazilian banking network is being reached by the increasingly global changes. Data Envelopment Analysis was applied to measure the competition level through corporate performance among Brazilian banks. We used data about total owner capital, liability, total revenue, and semester output of 144 banks. Banks were divided into three groups: big, medium, and small-sized banks. BCG matrix approach was used to classify the several operational efficiency and profitability indicators. The results show big banks have the best performance and small banks the worst performance.
\end{abstract}

Key words: performance; data envelopment analysis; banks sector. 


\section{INTRODUÇÃO}

O final do século passado foi marcado por acirrada concorrência em todos os setores da economia mundial. É natural que num setor competitivo apenas as organizações mais fortes sobrevivam. Assim, os administradores deverão estar constantemente perseguindo ganhos de eficiência sem se distanciarem de um aspecto financeiro básico, a rentabilidade do negócio.

No Brasil, o sistema bancário é caraterizado por diversas fusões e incorporações em vista de maior solidez das instituições financeiras. Além disso, está em pleno desenvolvimento um amplo processo de adaptação e expansão de modernas tecnologias de gestão que propiciam maior satisfação aos clientes, em nível tanto interno quanto externo das organizações. Essas tecnologias induzem a assimilação e a adaptação de novos paradigmas gerenciais existentes em outros países, enfatizando-se desde pequenos ajustes operacionais até a redefinição da estratégia de negócio das empresas. Essas tecnologias visam a tornar a empresa mais competitiva, com ganhos de eficiência e rentabilidade de longo prazo.

O objetivo deste trabalho é investigar, comparativamente, a posição competitiva de 144 conglomerados financeiros no setor bancário brasileiro por meio da matriz rentabilidade versus eficiência produtiva. Essa matriz é estruturada com base na análise por envoltória de dados (DEA, do inglês Data Envelopment Analysis). Foram utilizados dados sobre o volume de receita, resultado do semestre, capital próprio e capital de terceiros. Os conglomerados foram agrupados, segundo o seu porte, em três categorias: grandes, médios e pequenos. Foram utilizados somente dados referentes ao segundo semestre de 1999, todos de conhecimento público. Desse modo, os dados de caráter restrito associados às questões estratégicas não fazem parte do estudo. Este é um limitador que atinge todas as pesquisas empíricas realizadas sob a ótica do analista externo. Mesmo com esta restrição, o trabalho é de suma importância para que os administradores tenham uma visão do desempenho global e integrado de cada conglomerado.

O artigo está estruturado da seguinte maneira: na segunda seção, é realizada breve revisão dos modelos de análise de desempenho técnico em bancos; na terceira seção, descrevem-se alguns aspectos relacionados aos dados da amostra e à metodologia utilizada; na quarta seção, são apresentados os resultados obtidos; a quinta seção é reservada às conclusões. 


\section{Análise de Desempenho Técnico em Instituiçóes Bancárias}

São numerosas as técnicas quantitativas desenvolvidas para medir o desempenho técnico de um departamento dentro de uma empresa ou de uma empresa dentro de um setor qualquer. Existem técnicas de caráter qualitativo e comportamental, como a visão estratégica de negócio; mas essas não serão objeto deste artigo, porque são necessárias informações geralmente restritas a poucas pessoas em cada organização, não sendo, portanto, de conhecimento público e acessível ao analista externo.

Basicamente, as técnicas quantitativas podem ser classificadas em três grandes grupos: (1) índices financeiros; (2) análise de regressão e fronteira estocástica; e (3) análise por envoltória de dados. Uma excelente revisão dessas e de outras técnicas pode ser obtida no artigo de Berger e Humphrey (1997). A seguir, consta uma definição dos três grupos de técnicas.

\section{Índices Financeiros}

Os índices financeiros são excelente ferramenta de investigação da situação empresarial; porém o seu enfoque é parcial, ou seja, o índice de liquidez identifica a situação de liquidez e o índice de lucratividade identifica a situação referente à lucratividade. Dessa forma, a análise acaba por seguir o famoso princípio de René Descartes: dividir o objeto de estudo (a empresa) no maior número possível de partes, para que se possa ter uma solução adequada da situação; contudo fragmentar a empresa em partes impede a identificação da sua situação global, pois se ignoram a inter-relação e a interdependência das variáveis analisadas.

Para o setor bancário dos Estados Unidos, DeYoung (1998) apresentou evidências de que a aplicação de índices financeiros fornece um quadro unidimensional e incompleto da organização. Grande parte desta constatação é devida à dificuldade que existe em agregar os diversos índices num indicador da situação global que reflita a inter-relação e a interdependência das diversas variáveis. A análise, geralmente, é realizada comparando-se os índices financeiros da empresa com índices médios do setor ou com os da própria empresa em períodos anteriores. Como não ocorre inter-relação e interdependência nos cálculos dos índices, não é possível identificar um benchmarking específico para as empresas. 


\section{Análise de Regressão}

A análise de regressão procura identificar a eficiência por meio da relação entre os valores observados da empresa e uma combinação de valores agregados por ponderações médias da amostra (parâmetros). Esses parâmetros são os coeficientes estimados pela equação de regressão que melhor se ajusta aos dados. A eficiência propriamente dita é uma relação de distância entre a posição observada da empresa e sua posição sobre a linha de regressão. Um exemplo de equação de regressão é dado pela Equação [1].

$R T_{k} \quad{ }_{i 1}^{n} x_{i k} v_{i} \quad{ }_{k}$.

onde $R T$ é a receita total; o subscrito $k(k=1, \ldots, N)$ representa a empresa analisada; $x_{i}(i=1, \ldots, n)$ é a variável de produção; $v_{i}$ é o coeficiente que definirá um padrão médio para a amostra, sendo estimado pela equação; e $\varepsilon_{k}$ mede exatamente a distância da empresa $k$ até a linha da equação de regressão. Se $\varepsilon_{k}=0$, a empresa $k$ estará exatamente sobre a linha estimada.

\section{Fronteira Estocástica}

O modelo de fronteira estocástica apresenta, segundo Anderson, Lewis e Springer (1999), uma vantagem sobre o modelo linear clássico de regressão. Ele decompõe o termo de erro em erro aleatório e erro de ineficiência gerencial. Esse fato melhora a precisão dos parâmetros estimados pela equação. O modelo de fronteira estocástica foi primeiramente introduzido por Aigner, Lovell e Schmidt (1977) e Meeusen e Broeck (1977). Uma completa descrição dos avanços deste enfoque pode ser obtida no trabalho de Bauer (1990). Um modelo simples de fronteira estocástica é especificado pela Equação [2].

$R T_{k}{ }_{i 1}^{n} x_{i k} v_{i} \quad\left(p_{k} \quad q_{k}\right)$.

A notação da Equação [2] é similar à da Equação [1], o que as diferenciam são os termos $p_{k}$ e $q_{k}$. A seqüência $p_{k}(k=1, \ldots, N)$ é um número positivo de perturbação que captura a ineficiência gerencial, por definição $p_{k} \sim N\left(0, \sigma_{p}^{2}\right)$. A seqüência $q_{k}(k=1, \ldots, N)$ é o termo de perturbação que captura a medida de erro aleatório, por definição $q_{k} \sim \operatorname{IID}\left(0, \sigma^{2}\right)$. A estimação da Equação [2] é obtida pela maximização da função de máxima verossimilhança dada por [3]. 
$\ln L \quad \frac{N}{2} \ln \frac{2}{-} \quad N \ln \quad{ }_{k 1}^{N} \ln 1 \quad \frac{k}{2^{2}}{ }_{k 1}^{N} k$

onde $N$ é o tamanho da amostra; $\varepsilon_{k}$ é a soma das perturbações $p_{k}$ e $q_{k}$; $\sigma^{2}$ é a variância do termo de erro composto definida por $\sigma_{p}^{2}+\sigma_{q}^{2} ; \Phi$ representa a função de distribuição normal padrão, e $\lambda$ representa a assimetria do termo de erro composto, sendo definida como $\sigma_{p} / \sigma_{q}$.

\section{Análise por Envoltória de Dados}

A análise por envoltória de dados (DEA) foi primeiramente introduzida por Charnes, Cooper e Rhodes (1978) e posteriormente estendida por Banker, Charnes e Cooper (1984). A DEA é uma técnica que utiliza os princípios da programação matemática linear para medir o desempenho de uma unidade sob avaliação (setor, empresa etc.) relativa às demais unidades. A técnica identifica uma fronteira eficiente composta por todas as unidades com as melhores práticas observadas, o que, por sua vez, elimina a necessidade de se predefinir um desempenho padrão (média) contra o qual, geralmente, são avaliadas todas as unidades. Isso significa que a DEA não é paramétrica, ao passo que os métodos citados anteriormente os são. Essa é a diferença fundamental entre eles. O modelo básico DEA é apresentado pelas formulações [4] e [5]. Considere $N$ empresas, cada uma produzindo $m$ diferentes quantidades de outputs pela utilização de $n$ diferentes quantidades de inputs. Nesse contexto, uma empresa $k$ qualquer produz $y_{r k}$ quantidades de outputs, a partir da utilização de $x_{i r}$ quantidades de inputs.

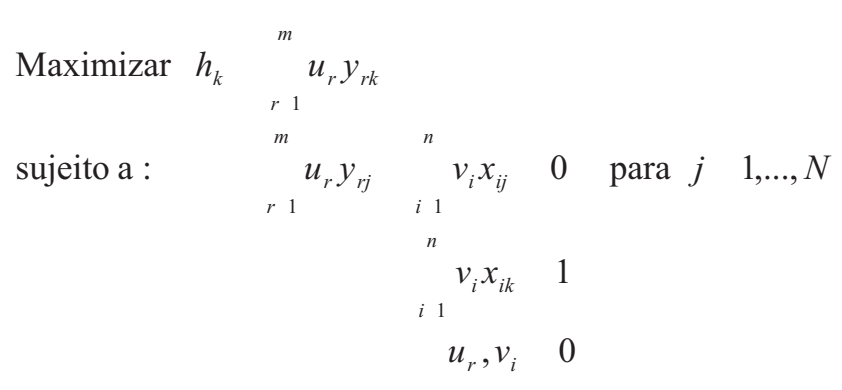

O objetivo em [4] é encontrar a máxima soma ponderada $h_{k}$, onde $u_{r}$ é o preço específico a ser encontrado para um output $r$, e $v_{i}$ o preço específico de cada input $i$. O indicador de eficiência da empresa $k$ é obtido pela razão entre a soma ponderada dos outputs e a soma ponderada dos inputs. 
Os preços $u_{r}$ e $v_{i}$ são determinados de maneira a maximizar a eficiência da empresa. Dessa forma, qualquer outro conjunto de pesos produzirá um indicador de eficiência inferior. A empresa será eficiente, se a razão for igual à unidade, e ineficiente se for menor do que a unidade. É importante ressaltar que nenhuma outra empresa da amostra poderá apresentar indicador de eficiência superior à unidade, se utilizar o conjunto de pesos da empresa analisada.

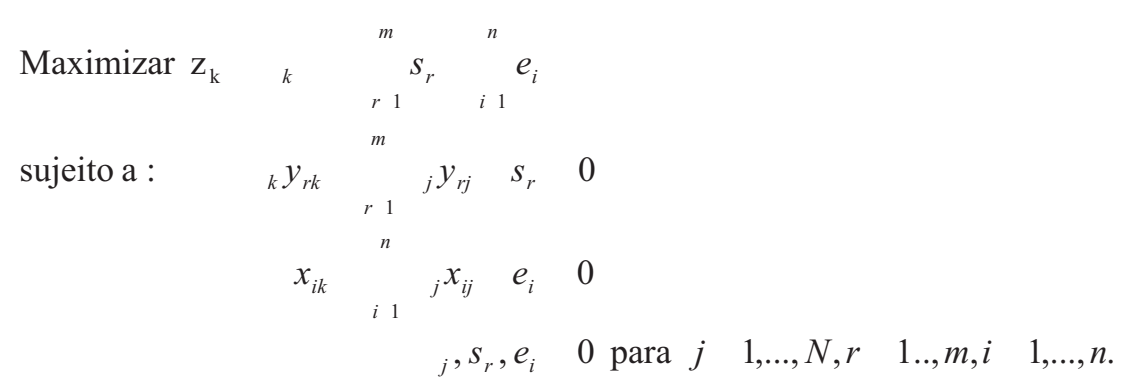

Na formulação [5], $z_{k}$ representa o aumento eqüiproporcional no nível de produção da empresa $k$, deslocando-a até a fronteira eficiente; $s_{r}$ e $e_{i}$ são, respectivamente, unidades deficitárias de output e excessivas de input; $\varepsilon$ é um valor infinitesimal positivo, geralmente tomado como $1 \times 10^{-6}$. Nota-se que foi utilizada a letra grega $\varepsilon$ para definir o infinitesimal $\varepsilon$ em [5] e $\varepsilon_{k}$ em [1] e [3]. Eles não guardam nenhuma relação, mas são tradicionalmente utilizados por ambas as literaturas, motivo pelo qual foram mantidos neste artigo. Já $\lambda_{j}$ é o vetor intensidade que identificará o(s) ponto(s) de comparação que repousa(m) sobre a fronteira. A empresa $k$ será dita eficiente, se o valor de $\theta_{k}$ for igual à unidade; e ineficiente se $\theta_{k}$ for maior que a unidade. Caso não exista qualquer restrição aos parâmetros de ponderação na formulação [4], ambas as formulações [4] e [5] apresentarão o mesmo indicador de eficiência.

Uma comparação gráfica entre a DEA e a equação de regressão é apresentada na Figura 1 . O eixo das ordenadas mede o valor de um único output, e o eixo das abcissas mede o valor de um único input. Cada ponto registrado nesta figura representa uma empresa. Observa-se que a linha de regressão identifica um nível médio de output para cada nível de input. Já a fronteira eficiente identifica as melhores práticas para cada nível de operação.

Na Figura 1, a empresa (a) tem o ponto (a'), identificado pela linha de regressão como sendo o que melhor representa o comportamento médio ao seu nível de operação. Para a empresa (b), o ponto de melhor desempenho médio é (b'); porém, nessa mesma situação, com a utilização da DEA, os pontos de referência são, respectivamente, (a") e (b"), os quais representam o comportamento de melhor prática observada para cada uma das empresas. 


\section{Figura 1: Comparação Gráfica entre Fronteira Eficiente e Linha de Regressão}

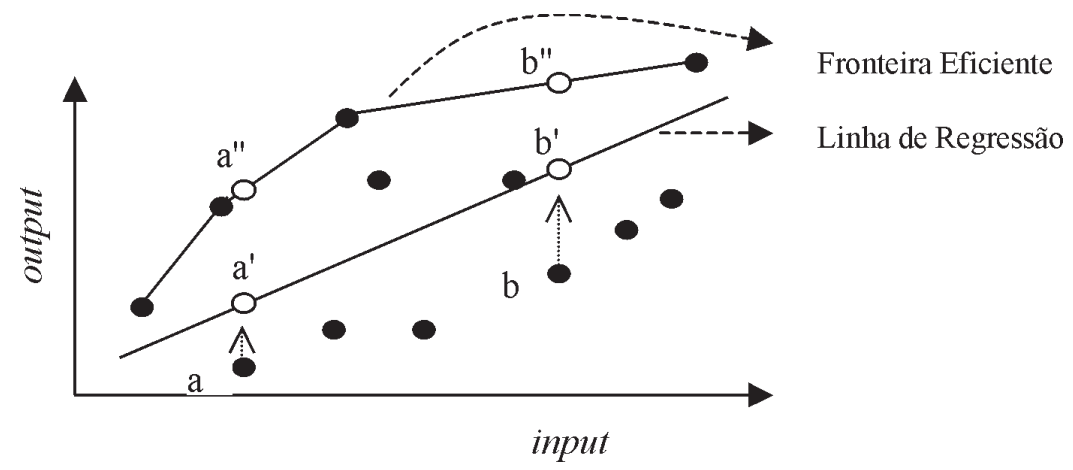

A diferença básica entre a DEA e a análise de regressão é que a primeira produz um indicador de eficiência que deriva da fronteira eficiente e que revela a fronteira de produção empírica de melhor prática. Para cada empresa ineficiente, que está abaixo da fronteira, a DEA identifica um ponto de referência específico, pelo qual ela será avaliada. Já a análise de regressão e o modelo estocástico são formulações paramétricas que necessitam de uma forma funcional especificada a priori (equação de regressão), relacionando-se a variável dependente com as variáveis independentes. $\mathrm{O}$ padrão de comparação é uma equação de comportamento médio, ou seja, uma equação que melhor se ajusta aos dados e que será utilizada para explicar o comportamento de cada uma das empresas.

É extensa a literatura que utiliza a DEA na avaliação do setor bancário. Alguns estudos mais recentes são os de Siems e Barr (1998), que investigam o setor bancário nos Estados Unidos; Zenios et al. (1999), que aplicam a DEA no Banco de Chipre; Ali e Chen (1999), que analisam o desempenho de 64 bancos internacionais, dos quais dois são brasileiros; Soteriou e Zenios (1999), que investigam o desempenho de bancos com uma abordagem integrada, e Althanassopoulos (1999), que direciona o estudo para uma abordagem integrada entre desempenho, qualidade e capacidade.

\section{Metodologia}

Os dados necessários para a realização deste trabalho foram obtidos no Banco Central do Brasil (BC). O BC disponibiliza, periodicamente, relatórios com diver- 
sas informações do setor bancário brasileiro (Relatório Banco Central, 19992000). Foram coletados dados para 144 conglomerados financeiros sobre o ativo circulante, exigibilidade, patrimônio líquido, receita total e resultado do semestre, relacionados ao segundo semestre de 1999. Como alternativa para isolar possíveis efeitos relacionados ao porte, os conglomerados foram agrupados nas categorias grandes, médios e pequenos, cada uma com, respectivamente, 16, 50 e 78 bancos. A Tabela 1 apresenta os valores médios para as três categorias, e a Tabela 2 alguns índices financeiros.

\section{Tabela 1: Valores Médios para as Categorias dos Conglomerados Financeiros, Relativos ao Segundo Semestre de 1999 (Valores em $\mathrm{R} \$ 1.000,00)$}

\begin{tabular}{lrrr}
\hline \multirow{2}{*}{ Contas } & \multicolumn{3}{c}{ Conglomerados Financeiros } \\
\cline { 2 - 4 } & \multicolumn{1}{c}{ Grandes } & \multicolumn{1}{c}{ Médios } & Pequenos \\
\hline Ativo Circulante + Realizável a Longo & & & \\
Prazo & $14.933 .897,00$ & $2.226 .190,00$ & $193.903,00$ \\
Ativo Permanente & $1.863 .284,00$ & $154.228,00$ & $13.879,00$ \\
Ativo Total & $16.797 .181,00$ & $2.380 .418,00$ & $207.781,00$ \\
Exigibilidades & $14.641 .650,00$ & $2.098 .128,00$ & $173.415,00$ \\
Patrimônio Líquido (a) & $2.105 .759,00$ & $271.399,00$ & $33.443,00$ \\
Receita Total & $3.113 .883,00$ & $577.944,00$ & $34.171,00$ \\
Resultado do Semestre & $49.773,00$ & $10.891,00$ & 923,00 \\
Observações (bancos) & 16 & 50 & 78 \\
\hline
\end{tabular}

(a) Não está computado o valor do resultado do semestre.

$\mathrm{Na}$ prática, além de tentar isolar possíveis efeitos relacionados ao porte, o agrupamento em categorias objetiva tornar o grupo mais homogêneo possível. A definição das categorias não seguiu nenhum rigor estatístico. Baseou-se apenas no valor decrescente do patrimônio líquido. Nas categorias de grande e médio porte, a relação entre o maior e o menor conglomerado é inferior a dez vezes; na categoria pequeno porte, é inferior a 14 vezes. Um critério interessante para definição de categorias é descrito em Zenios et al. (1999).

Observa-se, na Tabela 2, que a rentabilidade dos conglomerados financeiros de médio porte é superior a dos demais. Este fato pode induzir à conclusão de que eles têm melhor desempenho por proporcionar maior remuneração ao capital próprio. Por ora, essa conclusão é precipitada. Outra constatação é que a rentabilidade superior dos conglomerados de médio porte parece estar associada à 
alavancagem financeira (leverage), evidenciando que esses conglomerados estão adotando uma postura mais agressiva quanto à estrutura de capital.

\section{Tabela 2: Índices Financeiros ${ }^{(a)}$ para as Categorias dos Conglomerados Financeiros, Valores Relativos ao Segundo Semestre de 1999}

\begin{tabular}{lrrr}
\hline \multirow{2}{*}{ Indices } & \multicolumn{3}{c}{ Conglomerados Financerios } \\
\cline { 2 - 4 } & Grandes & Médios & Pequenos \\
\hline Rentabilidade (\%) (b) & 2,36 & 4,01 & 2,76 \\
Liquidez Geral & 1,02 & 1,06 & 1,12 \\
Alavancagem (c) & 6,95 & 7,73 & 5,19 \\
\hline
\end{tabular}

(a) Os índices financeiros foram calculados considerando-se os valores médios de cada categoria.

(b) A rentabilidade foi obtida dividindo-se o resultado do semestre pelo valor do patrimônio líquido.

(c) Para o cálculo do indicador de alavancagem, adicionou-se o resultado do semestre ao valor do patrimônio líquido (Alavancagem $=$ Exigibilidades $/$ Patrimônio Líquido).

Para investigar o desempenho de 144 conglomerados financeiros, foi utilizado o enfoque integrado entre eficiência operacional e rentabilidade por meio da matriz BCG (Hedley, 1976). Devido ao fato de os conglomerados terem sido agrupados em categorias, amenizando as diferenças de porte organizacional, foram utilizados modelos de eficiência operacional e de rentabilidade sob a hipótese de retornos constantes de escala. Com isso, assume-se que a relação de proporcionalidade entre output e input é mantida inalterada, independentemente do nível de operação. Esses modelos são operacionalizados com base na DEA e estão descritos a seguir.

\section{Modelo de Eficiência Operacional}

O modelo de eficiência operacional tem por objetivo investigar o desempenho relativo à combinação de recursos (capital próprio e de terceiros) para a obtenção de participação de mercado (receita total). É operacionalizado pela formulação [5]. Nesse caso, o conglomerado será considerado eficiente se $1 / z=1$, e ineficiente se $1 / z<1$. Observe que $z$ identifica o aumento eqüiproporcional no nível de produção, deslocando as empresas ineficientes até a fronteira, e $1 / z$ identifica o respectivo grau de eficiência. 


\section{Modelo de Rentabilidade}

O modelo de rentabilidade investiga o desempenho dos conglomerados referente à sua capacidade de remuneração do capital dos seus proprietários. É operacionalizado pela formulação [5] com ajuste para incorporar variáveis negativas (vide Apêndice).

\section{Análise Integrada}

A análise integrada é desenvolvida pela união dos modelos de eficiência operacional e rentabilidade, mediante a matriz BCG. Essa matriz é formada por quatro quadrantes denominados de Stars, Dogs, Sleepers e Cows. O quadrante Stars engloba organizações que apresentam desempenho superior em termos de eficiência operacional e rentabilidade. Cows são as organizações eficientes, mas que apresentam baixa rentabilidade, revelando organizações influenciadas por aspectos negativos do ambiente econômico. Sleepers são organizações que apresentam elevada rentabilidade, mas são ineficientes, estando a rentabilidade associada mais às questões do ambiente econômico do que a aspectos gerenciais. $\mathrm{O}$ quadrante Dogs é uma situação difícil para as organizações. A presença nesse quadrante é indesejável, pois engloba ineficiência gerencial e desfavorecimento do ambiente econômico.

\section{Resultados Obtidos}

Os resultados obtidos pela aplicação dos modelos de eficiência operacional e de rentabilidade são apresentados de forma resumida na Tabela 3. Na primeira coluna estão listadas as estatísticas de ambos os modelos. Nas colunas seguintes são apresentados os diversos valores médios obtidos para cada uma das três categorias bancárias.

Com base nos valores apresentados na Tabela 3, é possível afirmar que os conglomerados de grande porte têm grau de eficiência operacional muito superior aos de médio porte e estes em relação ao de pequeno porte, tanto em relação ao valor médio quanto ao valor mínimo. Relativamente ao modelo de rentabilidade, os conglomerados de grande porte também têm eficiência superior a dos demais. Já os de médio porte apresentam eficiência semelhante aos de pequeno porte. 
Tabela 3: Estatística Descritiva dos Indicadores de Eficiência Operacional e de Rentabilidade de 144 Conglomerados Financeiros

\begin{tabular}{lccc}
\hline \multirow{2}{*}{ Estatística Descritiva } & \multicolumn{3}{c}{ Conglomerados Financeiros } \\
\cline { 2 - 4 } & Grandes & Médios & Pequenos \\
\hline Modelo de eficiência operacional & 0,78 & 0,50 & 0,40 \\
Média & 1,00 & 1,00 & 1,00 \\
Máximo & 0,40 & 0,18 & 0,11 \\
Mínimo & 0,20 & 0,24 & 0,21 \\
Desvio Padrão & & & \\
Modelo de rentabilidade & 0,21 & 0,05 & 0,06 \\
Média & 1,00 & 1,00 & 1,00 \\
Máximo & $-1,75$ & $-0,38$ & $-0,52$ \\
Mínimo & 0,66 & 0,20 & 0,19 \\
Desvio Padrão &
\end{tabular}

Nota: o valor 1,00 representa $100 \%$ de eficiência; logo, o valor 0,78 é interpretado como $78 \%$ de eficiência.

Fato importante é a grande diferença que existe entre os resultados do modelo de rentabilidade com os valores apresentados na Tabela 2. Nessa tabela os resultados induziam a pensar que os conglomerados de melhor desempenho eram os de médio porte com rentabilidade média de $4,01 \%$, seguidos pelos de pequeno porte $(2,76 \%)$ e os de grande porte $(2,36 \%)$. Já na Tabela 3, os mais eficientes são os de grande porte (21\%), seguidos pelos de pequeno porte $(6 \%)$ e os de médio porte (5\%). A diferença entre os resultados é devida ao agrupamento em categorias que, na análise DEA, ameniza os efeitos do porte organizacional e o confronto entre organizações não homogêneas.

As Figuras 2, 3 e 4 (matriz BCG) possibilitam uma análise integrada dos indicadores de eficiência técnica e de rentabilidade para os conglomerados nas três categorias. No eixo das ordenadas, são apresentados os indicadores de rentabilidade e, no eixo da abcissas, os indicadores de eficiência operacional. Cada ponto identifica o desempenho de um conglomerado. Não há dúvida de que o melhor para os conglomerados é estarem representados no quadrante Stars; contudo os quadrantes Sleeper e Cows também são aceitáveis; porém o mesmo não pode ser afirmado para o quadrante Dogs (para efeitos visuais, foi suprimido da Figura 2, o conglomerado que apresentou indicador de rentabilidade -1,75 e, da Figura 4, o que apresentou indicador de rentabilidade de -0,52).

Figura 2: Rentabilidade e Eficiência Operacional para Conglomerados 
Financeiros de Grande Porte

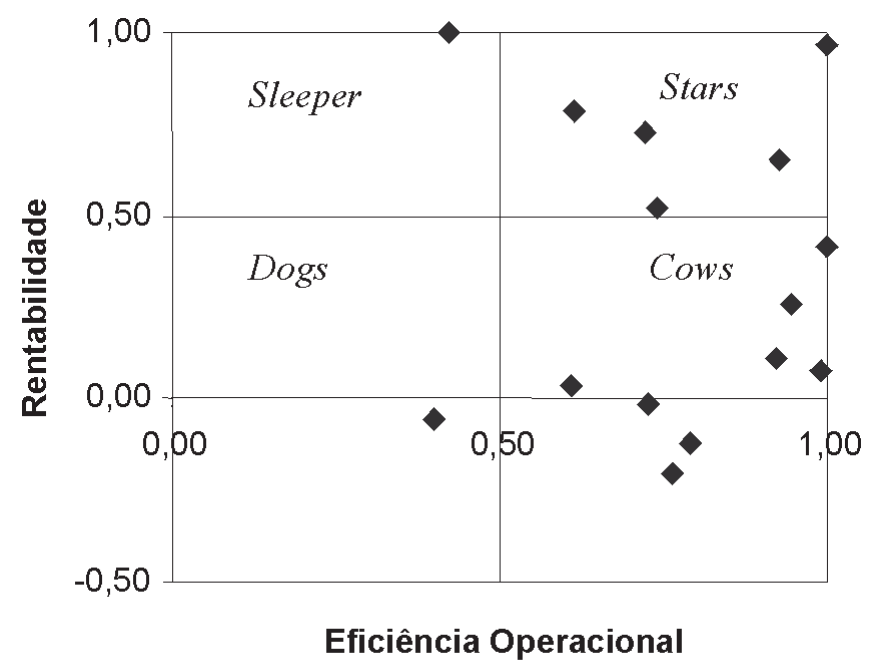

Figura 3: Rentabilidade e Eficiência Operacional para Conglomerados Financeiros de Médio Porte

Figura 4: Rentabilidade e Eficiência Operacional para Conglomerados

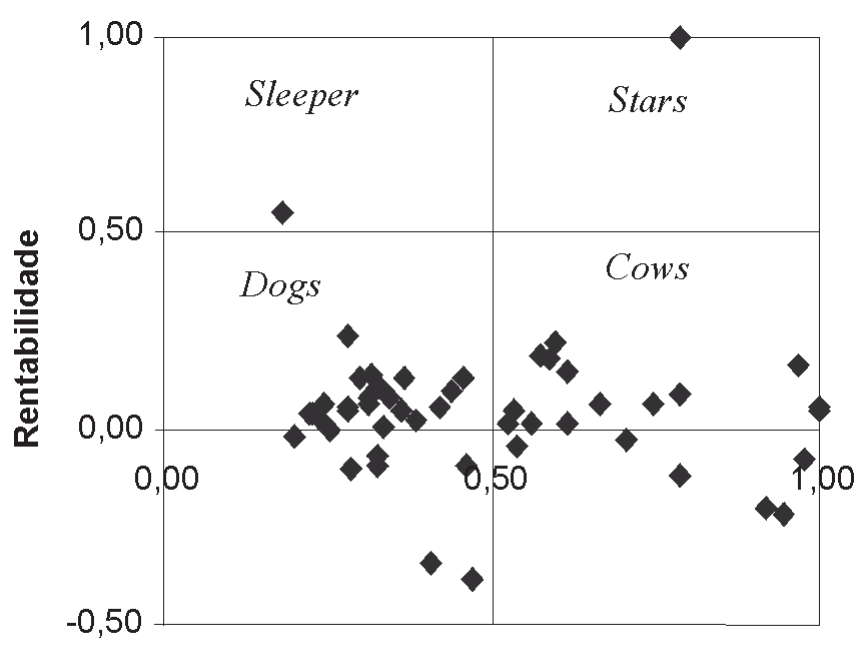

Eficiência Operacional 


\section{Financeiros de Pequeno Porte}

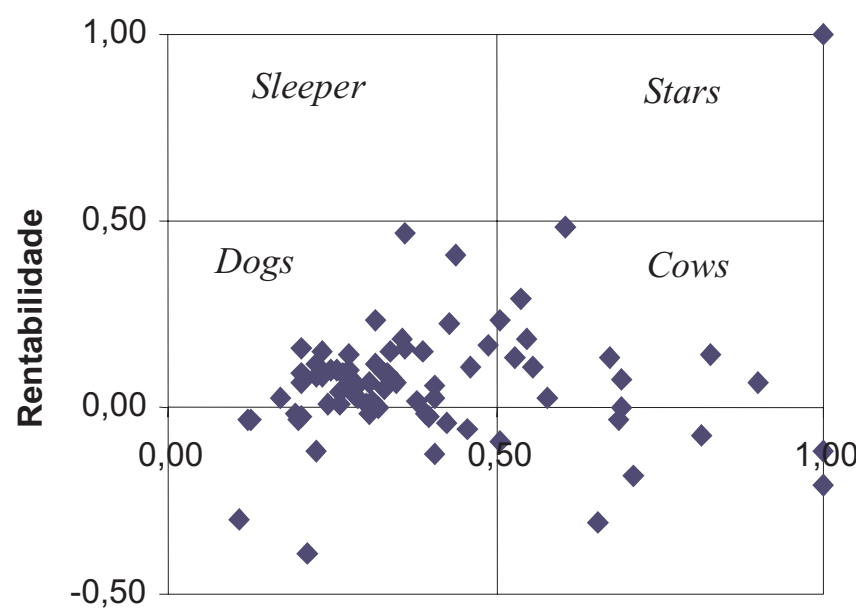

Eficiência Operacional

Pela observação visual da matriz BCG, é fácil constatar que os conglomerados financeiros de grande porte apresentam desempenho superior aos demais, pois $31 \%$ (5 conglomerados) estão localizados no quadrante Stars. Bem diferente é a situação dos conglomerados de médio porte. Apenas um banco está no quadrante Stars e, aproximadamente, $50 \%$ na situação indesejável do quadrante Dogs. Pior ainda é a situação dos conglomerados de pequeno porte, onde a grande maioria está representada no quadrante Dogs e apenas um banco no quadrante Stars. Também é possível afirmar que existe pouca relação entre os indicadores de eficiência operacional e de rentabilidade, pois não há evidências de associação direta ou indireta entre eles.

As Figuras 5, 6 e 7 ilustram de maneira simples a ineficiência em unidades de output, ou seja, os valores deficitários de receita correspondente a cada um dos conglomerados. O eixo vertical mede a receita em milhões de reais, enquanto os bancos são identificados no eixo horizontal. As unidades deficitárias são a diferença entre a receita ideal e a receita atual. A receita ideal é obtida pela multiplicação da receita atual pelo valor de $z$ no modelo [5]. 
Figura 5: Déficit de Receita para os Conglomerados de Grande Porte

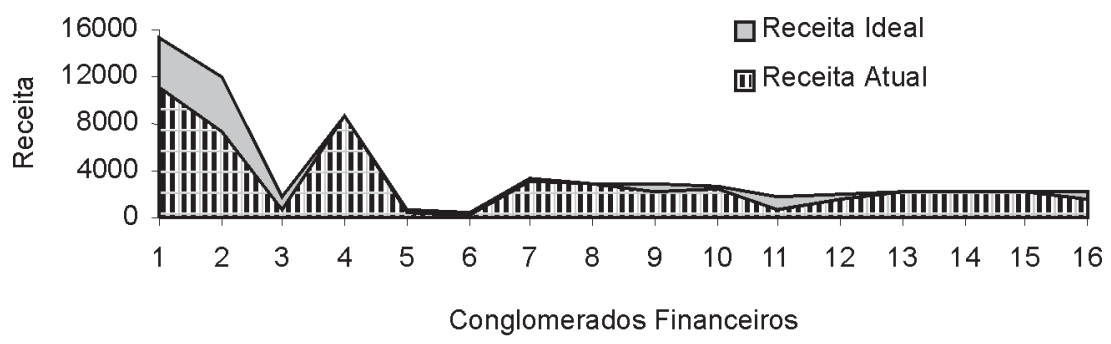

Figura 6: Déficit de Receita para os Conglomerados de Médio Porte

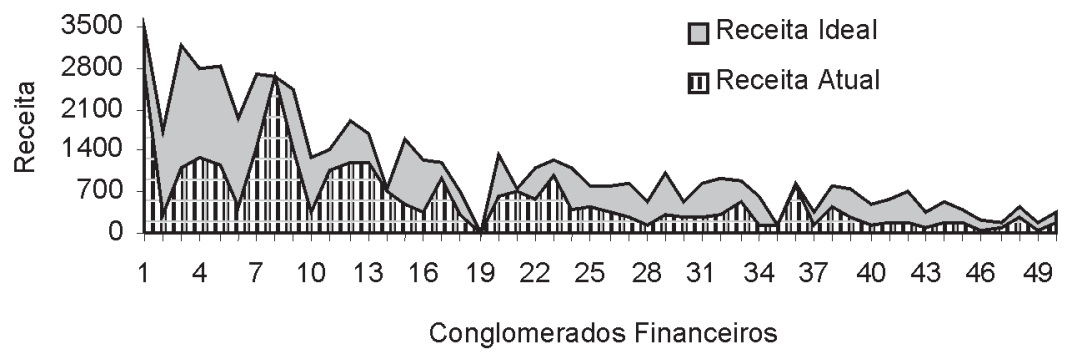

Figura 7: Déficit de Receita para os Conglomerados de Pequeno Porte

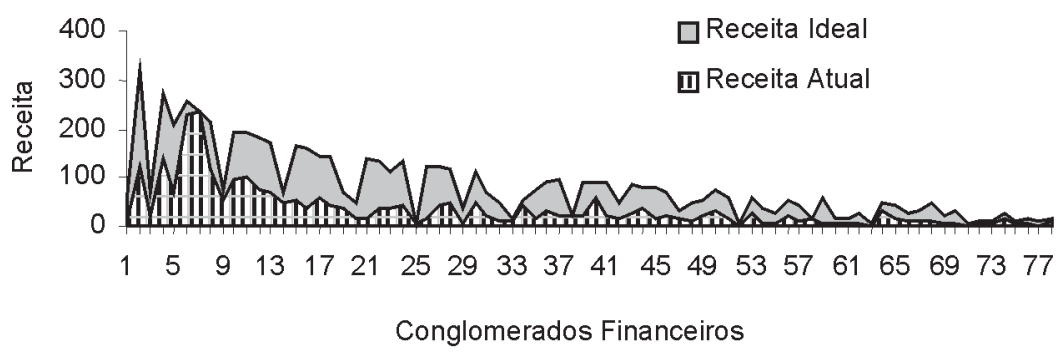

A observação visual das Figuras 5, 6 e 7 confirma que os conglomerados de grande porte são os que apresentam a menor quantidade de ineficiência operacional, não havendo muita discrepância entre o nível de receitas ideal e atual.

De forma idêntica, as Figuras 8, 9 e 10 ilustram a ineficiência em unidades relativas ao resultado do semestre para cada um dos conglomerados. $\mathrm{O}$ eixo vertical mede o valor do resultado do semestre em milhões de reais. O eixo horizontal identifica os conglomerados. As unidades deficitárias são a diferença entre o resultado ideal e o resultado real. 
Figura 8: Déficit de Resultado do Semestre para os Conglomerados de Grande Porte

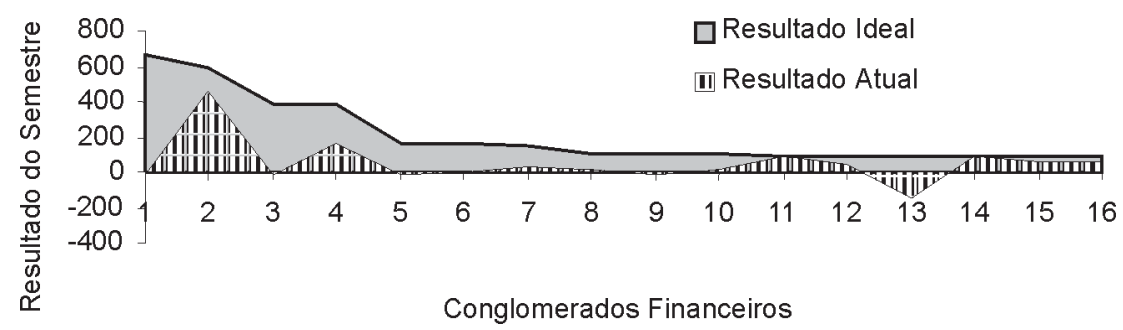

Figura 9: Déficit de Resultado do Semestre para os Conglomerados de Médio Porte

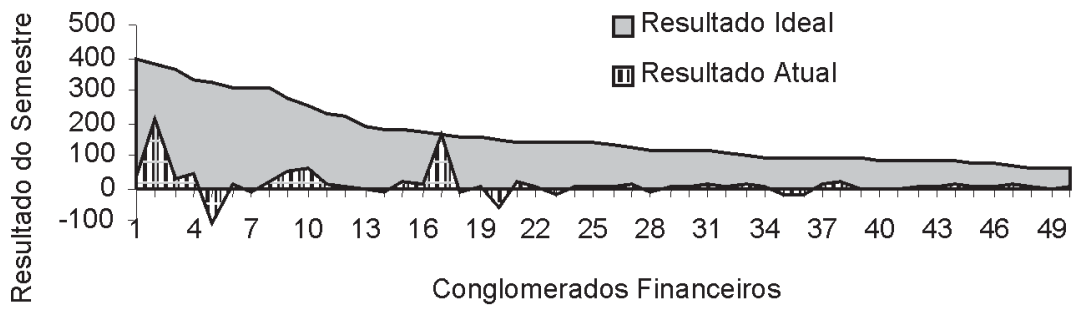

\section{Figura 10: Déficit de Resultado do Semestre para os Conglomerados de Grande Porte}

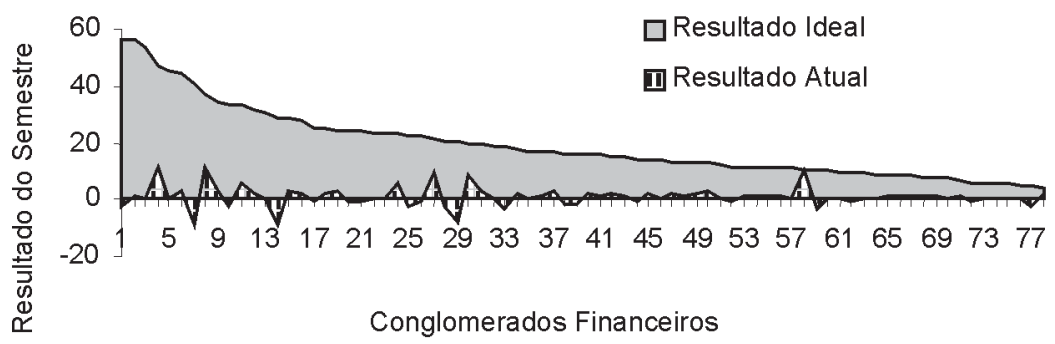

Nas Figuras 8, 9 e 10 o resultado ideal é obtido pela multiplicação do resultado atual pelo valor de $z$ em [7], que está no Apêndice. Nesse enfoque, os conglomerados financeiros de grande porte apresentam elevado grau de ineficiência; mas, mesmo assim, estão em situação favorável em relação aos de médio e pequeno porte.

Por fim, as Figuras 11, 12 e 13 ilustram o excesso de exigibilidade que deve ser eliminado mesmo se os conglomerados passassem a atuar nos níveis de receita 
(Figuras 5,6 e 7) e do resultado de semestre (Figuras 8, 9 e 10). O eixo vertical mede o valor da exigibilidade em milhões de reais. $\mathrm{O}$ valor excessivo de exigibilidade é a diferença entre a exigibilidade atual e a ideal.

Figura 11: Excesso de Exigibilidade nos Conglomerados de Grande Porte

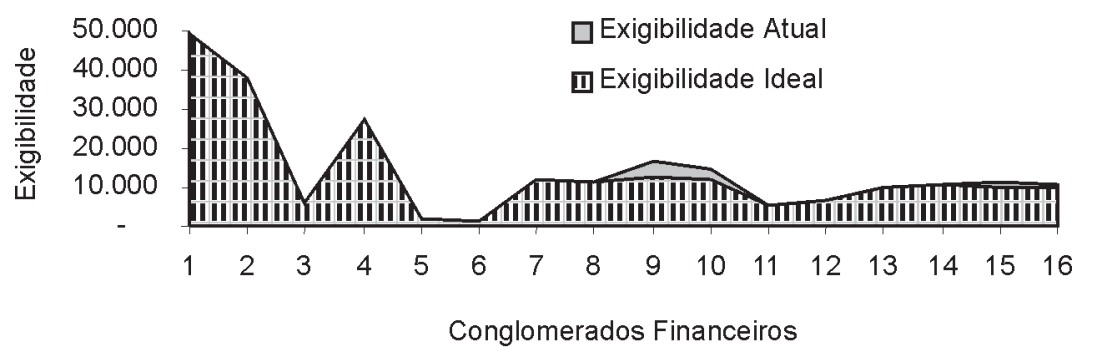

Figura 12: Excesso de Exigibilidade nos Conglomerados de Médio Porte

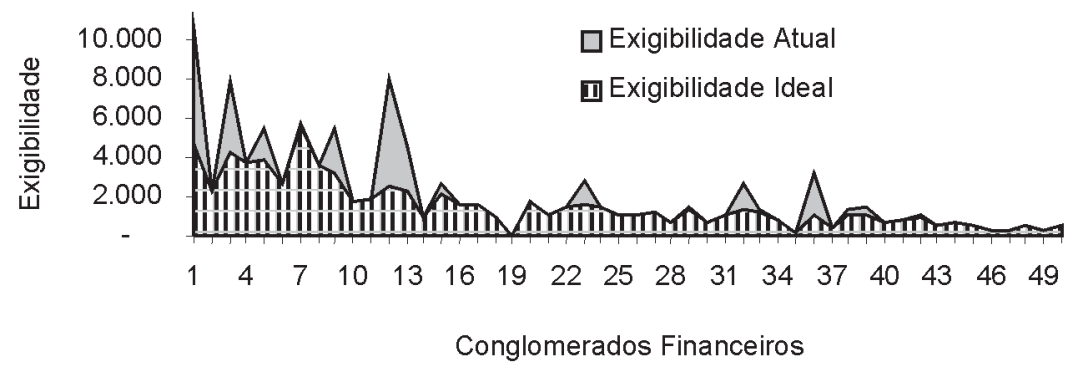

Figura 13: Excesso de Exigibilidade nos Conglomerados de Pequeno Porte

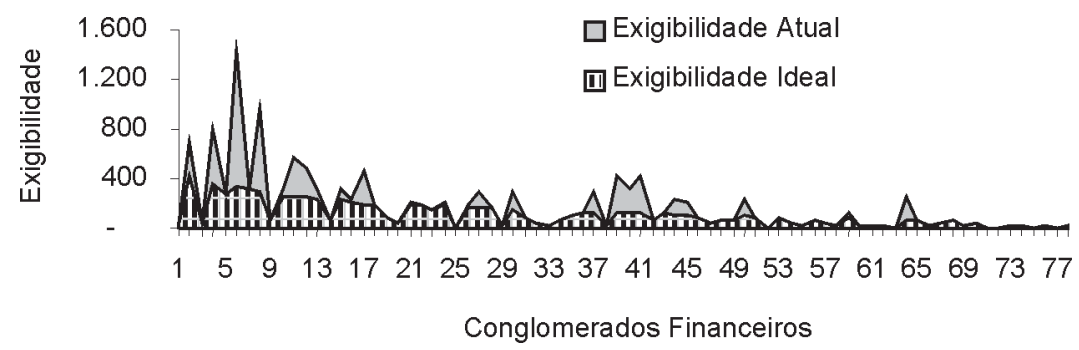

Semelhantemente às situações anteriores, os conglomerados de grande porte são os que apresentam a melhor harmonia no uso de capital próprio e de terceiros, enquanto os de médio e pequeno porte tendem a atuar com uma estrutura com grandes excessos de capital de terceiros. 


\section{CONClusão}

Este artigo investigou o desempenho integrado de 144 conglomerados financeiros, por meio da aplicação de dois modelos distintos de DEA, o modelo de eficiência operacional e o modelo de rentabilidade. Foram utilizados dados sobre o montante de capital próprio, capital de terceiros, receita total e resultado do semestre. A análise integrada dos indicadores de eficiência permite concluir que os conglomerados de grande porte são os de melhor desempenho, com a maioria de seus representantes operando nos quadrantes aceitáveis da matriz BCG. Por outro lado, a categoria de pequeno porte apresentou a maioria de seus conglomerados operando no quadrante indesejável de baixa rentabilidade e baixa eficiência operacional. Essas constatações parecem refletir o que vem ocorrendo neste setor, com a falência de pequenas e médias instituições bancárias e mesmo a compra ou fusão entre bancos nacionais e estrangeiros. Como já ocorreu em outros setores, o mercado caminha para um número menor de instituições bancárias, mas de grande porte.

\section{Referencias Bibliográficas}

AIGNER, D.;

LOVELL, C. A.;

SCHMIDT, P.

Formulation and estimation of stochastic frontier function models. Journal of Econometrics, v. 6, p. 21-37, 1977.

ALI, A. I.;

CHEN, Y.

Benchmark data envelopment analysis with an application to fortune global 500 banks. Working Paper, The University of Massachusetts, 1999.

\section{ALTHANASSOPOULOS, A.}

An optimization framework for the triad: capabilities, service quality and performance. In: HARKER, P. T.; ZENIOS, S.A. Performance of financial institutions. Cambridge: Cambridge University Press, 1999.

ANDERSON, R. I.;

LEWIS, D.;

SPRINGER, T. M.

Operating efficiencies in real estate: a critical review of the literature. Journal of Real Estate Literature, v. 8, n. 1, p. 3-18, 1999. 
BANKER, R. D.;

CHARNES, A.;

COOPER, W. W.

Models for the estimation of technical and scale efficiencies in data envelopment analysis. Management Science, v. 30, p. 1078-1092, 1984.

BAUER, P. W.

Recent developments in the econometric estimation of frontier. Journal of Econometrics, v. 46, p. 39-56, 1990.

BERGER, A. N.;

HUMPHREY, D. B.

Efficiency of financial institutions: international survey and directions for future research. European Journal of Operational Research, v. 98, p. 175-212, 1997.

CHARNES, A.;

COOPER, W. W.;

RHODES, E.

Measuring efficiency of decision making units. European Journal of Operational Research, v. 2, p. 429-444, 1978.

DEYOUNG, R.

Management quality and $\mathrm{x}$-inefficiency in national banks. Journal of Financial Services Research, v. 13, p. 5-22, Feb. 1998.
HEDLEY, B.

A fundamental approach to strategy development. Long Range Planning, [s.n.], p. 2-11, Dec. 1976.

MEEUSEN, W.;

BROECK, V. D.

Efficiency estimation from cobbdouglas production function with composed error. International Economic Review, v. 18, p. 435444, 1977.

PASTOR, J, T.

Translation invariance in data envelopment analysis: a generalization. Annals of Operations Research, v. 66, p. 93-112, 1996.

RELATÓRIO BANCO CENTRAL.

Banco Central do Brasil, 1999-2000.

SHAFER, S.;

BYRD, T. A.

A framework for measuring the efficiency of organizational investments in information technology using data envelopment analysis. Omega, v. 28, p. 125-141, 2000.

SIEMS, T. F.;

BARR, R. S.

Benchmarking the productive efficiency of U.S. banks. Financial Industry Studies, [s.n.], p. 11-24, Dec. 1998. 
SOTERIOU, A.;

ZENIOS, S. A.

Operations, quality and profitability in the provision of banking services. Management Science, v. 45, n. 9, p. 1221-1238, 1999.
ZENIOS, C. V. et al.

Benchmarks of the efficiency of bank branches. Interfaces, v. 29, n. 3, p. 37-51, 1999. 


\section{APÊNDICE}

Tradicionalmente, na literatura especializada, a análise por envoltória de dados é aplicada somente a valores positivos; porém, como enfatizam Pastor (1996) e Shafer e Byrd (2000), pode ser utilizada em situações que apresentem valores negativos, desde que seja realizada uma conversão nos dados, tornando-os positivos e sem prejudicar a solução do problema. Neste trabalho, foi desenvolvida uma modificação no modelo original DEA, tornando possível a sua aplicação em situações que apresentem valores negativos, sem a necessidade de convertê-los em valores positivos. O modelo é aplicável apenas em situações em que as empresas que apresentam outputs negativos não sejam utilizadas como referenciais de comparação, ou seja, que elas não participem da fronteira eficiente. A nova formulação é dada por:

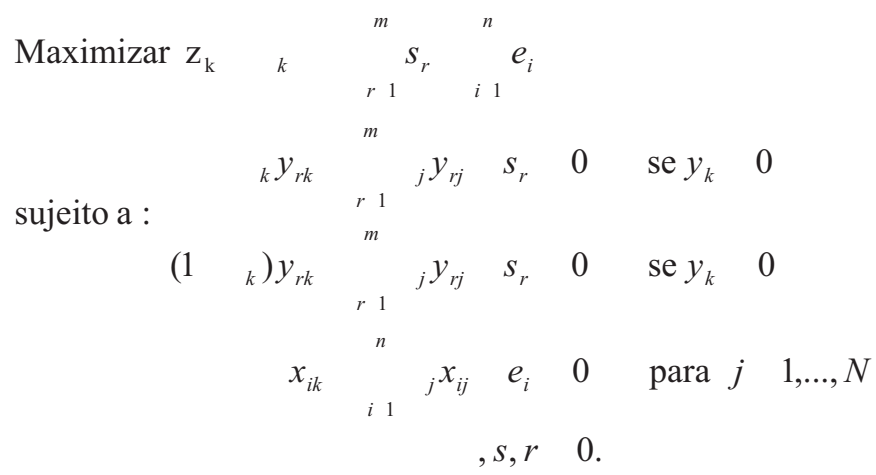

O indicador de eficiência da empresa $k$ é dado pela seguinte equação geral:

$\mathrm{Ef}_{\mathrm{k}}=\frac{\frac{p}{(n} \frac{n}{(1)}}{p \quad}$

onde $p$ é o número de outputs positivos, e $n$ é o número de outputs negativos de $k$.

A projeção da empresa para a fronteira eficiente é dada por:

1) Multiplicar os outputs positivos por $\theta$;

2) Multiplicar os outputs negativos por (1- $\theta)$. 